\title{
Enhancing Nigerian Healthcare Providers Competence in Breast Cancer Clinical Pathway Development using Blended Learning Approach
}

\author{
Kelechi Eguzo $^{1}$, Usenime Akpanudo ${ }^{2}$, Chukwuemeka Oluoha ${ }^{3}$, Nofisat Ismaila ${ }^{4}$, \\ Aniekan Jacob ${ }^{1}$, Kingsley Nnah ${ }^{1}$, Nuhu Tumba ${ }^{5}$, Mfonobong Udoekong ${ }^{6}$, Charles \\ Adisa $^{2}$
}

${ }^{1}$ Marjorie Bash Foundation/Marjorie Bash College of Health Sciences and Technology, Aba, Abia State, Nigeria. ${ }^{2}$ Harding University, Canada. ${ }^{3}$ Abia State University, Nigeria. ${ }^{4}$ American Society of Clinical Oncology, United States. ${ }^{5}$ Ahmadu Bello University Teaching Hospital, Nigeria. ${ }^{6}$ Obong University, Nigeria.

\begin{abstract}
Purpose: Clinical pathways are a means of translating universal clinical guidelines into local protocols to inform clinical practice. This study aimed to evaluate the effectiveness of using a blended learning approach for providing instruction and building competency on the development of breast cancer clinical pathways, using the guidelines from the National Cancer Control Network (NCCN). Methods: A one-group pretest-post test design was used for this study. Participants included healthcare providers and students in Nigeria, who were recruited through social media and professional organizations. The intervention was an online course delivered through Google Classroom, followed by two-day workshops at three locations. Data collection involved pre and post-tests scores from the online course, self-reported evaluation, as well as objective grading of in-person group projects. Results: Over 400 individuals joined the online course while 90 participated in the in-person workshops. Most participants $(259 / 408,63 \%)$ had no prior experience with online education, with an average age of 27.46 $( \pm 9.25)$ years. Data analysis revealed significant increases in mean knowledge scores across all groups from pretest $(\mathrm{M}=12.76, \mathrm{SE}=0.60)$ to post test $(\mathrm{M}=17.25, \mathrm{SE}=0.38) \mathrm{p}<0.001$. Self-reported evaluation showed higher scores regarding chemotherapy administration for blended learning participants. Objective competence scores in developing clinical pathways ranged from $63 \%$ to $87 \%$, across three sites. Conclusion: Findings reveal that both an online course alongside in-person workshops can be effective strategies for improving the knowledge and competence respectively of healthcare professionals on clinical pathways development. Participants in the blended learning component demonstrated significantly greater improvement in self-reported confidence scores. We recommend further studies to compare the relative strengths and limitations of these strategies.
\end{abstract}

Keywords: Breast cancer-blended learning-continuing medical education-online education-Nigeria

Asian Pac J Cancer Care, 6 (3), 271-276
Submission Date: 04/10/2021Ａcceptance Date: 06/16/2021

\section{Introduction}

Clinical pathways (CPs) are evidence-based multidisciplinary care plans that outline the essential steps needed in providing care for patients with specific clinical conditions (e.g. breast cancer). Essentially, CPs are a means of translating universal clinical guidelines into local protocols to inform clinical practice. These care plans are critical for linking the wealth of empirical evidence for specific health conditions into basic rule guides for clinical practice. When properly developed and implemented, CPs optimize patient outcomes and maximize clinical efficiency [1-2]. In the treatment of breast cancer, the use of CPs has been shown to reduce hospitalization, improve patient care process, reduce patient anxiety [3], and improve overall patient satisfaction [4]. Surprisingly,

Corresponding Author:

Dr. Kelechi Eguzo

Marjorie Bash Foundation/Marjorie Bash College of Health Sciences and Technology, Aba, Abia State - Nigeria.

Email:keguzo@gmail.com 
although breast cancer is the most common malignancy affecting women in Nigerian [5-6] the use of clinical pathways (CPs) for the management of breast cancer is not common practice in Nigerian hospitals [7].

Report from an ASCO-sponsored course in Abia State [7] revealed that physicians and nurses in the area were not fully confident about the effectiveness of their current strategies for optimizing local resources to match the requirements of international cancer management guidelines. Given this situation, simplifying complex guidelines into local CPs might improve the use of breast cancer clinical guidelines in Abia State, and the entire country (Nigeria). There is, however, no existing data regarding the skills of local multidisciplinary teams on the development of CPs in Nigeria making it impossible to draw empirically grounded conclusions about the effectiveness of such in the local Nigerian context.

In the extant literature, the blend of online and in-person training (i.e. blended learning) has been proposed as an effective and efficient means of improving the knowledge and competence of healthcare providers especially when traditional face-to-face strategies are not feasible [8-11]. Blended learning follows the tradition of self-directed learning in continuing medical education and involves the use of electronic and face-to-face classes to teach clinical concepts. This approach has been found to be an effective means of continuing education in several clinical areas including family medicine, orthopedics, and undergraduate medical education.[11] There had been no previous research that explored the impact of the blended learning approach regarding cancer education.

The purpose of the current investigation was to explore the effectiveness of using online and face-to-face strategies for providing instruction to improve knowledge and building competency on the development of clinical pathways for the management and treatment of breast cancer among healthcare providers in Nigeria. To address this purpose, the following research questions (RQ) were developed:

RQ1: To what extent does an online course improve participants' knowledge of clinical guidelines and pathways for breast cancer management?

RQ2: To what extent does an in-person workshop help participants gain competence in developing local CPs for breast cancer management?

\section{Materials and Methods}

This study used a one-group pretest-post test design to evaluate the effectiveness of using a combination of online learning and workshop approaches in the training of healthcare professionals and students. Participants from all the six geopolitical zones of Nigeria were recruited for the project using social media (Facebook, Twitter, and WhatsApp), text messaging, and through relevant professional organizations. Solicitation went out to physicians, nurses, and students enrolled in healthcare programs (e.g. medicine and nursing). Interested individuals were required to self-enroll online in order to participate in the study. This study was part of a larger project to provide professional development training for breast cancer management, using blended learning methodology. A minimum sample size of 100 is required to identify a small effect size between participation in the course and objective improvements in knowledge regarding antimicrobials stewardship ( $\mathrm{r} 2=0.12$, with power of $90 \%$ and alpha of 0.05 ) [12].

\section{Intervention}

The intervention was in two parts. The first part consisted of a six-week online course that focused on breast cancer guidelines and clinical pathway development delivered using Google Classroom ${ }^{\circledR}$. As part of the online course, participants were instructed about breast cancer guidelines using the NCCN Harmonized Guideline for Sub-Saharan Africa. Content on steps to develop clinical pathways was adapted from the Cancer Institute of New South Wales. During the online course, participants were reminded periodically to complete assigned tasks, such as quizzes. The quizzes were self-grading, such that participants obtained their quiz scores upon submission. The quizzes were formatted in a way that question and answer options were shuffled automatically. This was aimed at reducing the potential for cheating among course participants.

The online course was followed by a series of two-day in-person workshops. The workshops were held at three locations (Aba, Obong Ntak and Benin City. At each of the locations, one day was devoted to revising the key concepts that were taught during the online component of the course. Participants also worked in groups to develop local CPs using a worksheet that was adapted from the Cancer Council of New South Wales [13]. Details of the project plan has been published as a research protocol [14].

\section{Data collection}

Data collection for the online course comprised scores from online tests that were administered before and after each module (i.e. pre-test and post-tests). These were linked to the content of the modules. Comparisons between quiz performance at the beginning and at the end gave a measure of the change in knowledge for each module. The pre-course survey, which provided demographic data (e.g. age, sex, previous online course experience) was modified from the instrument developed by Gonzalez et al [10]. They had studied the impact of a breast cancer course in Mexico. The modification was informed by the literature on the subject [15-17]. Some components of the post-survey were adapted from evaluation forms developed by the American Society for Clinical Oncology [7]. These surveys were pretested on a sample of the potential participants for clarity and ease of completion. Data collection for the face-to-face workshops came from the results of the group activities (development of clinical pathways) that were graded using a rubric (Table 1). The rubric was developed using the steps outlined by Cancer Institute NSW [13], including stakeholder mapping, data to support pathway, action plan, and dissemination plan. 


\section{Data Analysis}

Data analyses for this study involved descriptive and inferential strategies. Inferential strategies involved the use of mixed factorial analysis of variance (ANOVA) to compare pretest and post test performance among the groups of participants (students, physicians, nurses, and others) on the online course (RQ1). The analysis for RQ1 only considered individuals who completed all the eight quiz assignments in the course, as a varying number of people completed different quizzes. Descriptive techniques were used to explore the participants' performance in face-to-face workshops (RQ2). SPSS Version 26 was used for data analysis.

Ethics approval for this investigation was obtained from Abia State Ministry of Health, Umuahia, Nigeria (AB/MH/EDHR/1/18/01). All participants in the study were informed of the study objectives, possible risks, benefits, as well as their right to withdraw from the study at any time without penalty.

\section{Results}

Over $400(n=408)$ individuals self-registered for the breast cancer guidelines online training course. The mean age of participants in the online course was $27.46( \pm 9.25)$ years, with a range of 18 to 54 years. Table 2 provides a summary of the demographic characteristics of the subjects who participated in the online course module for clinical pathway development. Of this number, 51 participants completed the pretests and post-tests for all the modules.

\section{RQ1: Knowledge of Guidelines for Clinical Pathway Development}

To address this research question, we conducted a mixed factorial ANOVA to explore the effect of the online training on participants' knowledge of breast cancer management as it relates to developing CPs that are aligned with NCCN guidelines. This analysis considered only individuals who completed all the eight pretests and post-tests in the online course $(n=51)$, and not everyone who had participated in the course. Before conducting the analysis, the assumption of the equality of covariance matrices across the groups was examined using Box's M. Results of this analysis $\mathrm{F}(6,6935.21)=2.05, \mathrm{p}=0.056$

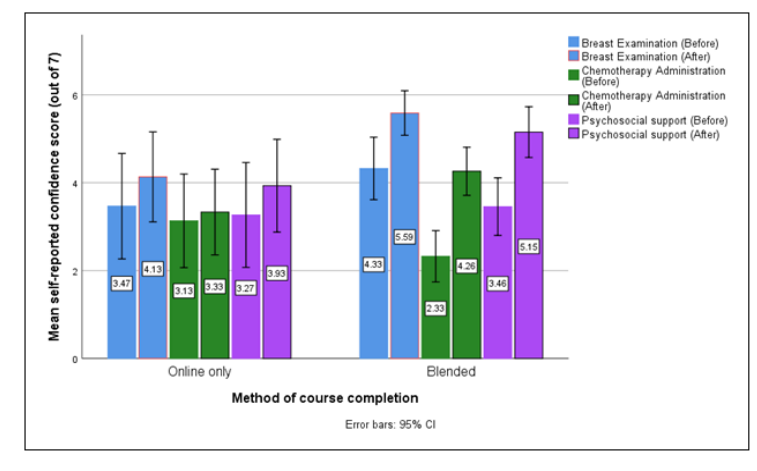

Figure 1. Comparing Self-reported Confidence Scores in Course Focus Areas

reveal that this assumption was met for the data. Table 3 provides descriptive statistics of the pretest and post test performance across sub-groups.

Results of the mixed factorial ANOVA analysis revealed that the interaction between participants' roles and their mastery of the knowledge related to developing CPs for breast cancer management as delivered in module 4 of the blended learning course was not statistically significant $F(3,47)=0.76, p=.520, \eta 2=.05$, a very small effect size. (18) On the other hand, the main effect for knowledge of developing CPs for breast cancer management was statistically significant $F(1,47)=63.01$, $\mathrm{p}<.001, \eta 2=.57$ (a larger than typical effect size). These results showed that for all participants, the difference between their estimated marginal mean pretest scores $(\mathrm{M}=12.76, \mathrm{SE}=0.60)$ and their mean post test scores $(\mathrm{M}=17.25, \mathrm{SE}=0.38)$ was large enough to be considered as representing a real difference in the population.

The main effect for participants' roles was not statistically significant $F(3,47)=1.01, p=.396, \eta 2=0.06$ (a smaller than typical effect size). These results revealed that the marginal means of breast cancer treatment knowledge scores (across both the pretest and post test) for students $(\mathrm{M}=14.89, \mathrm{SE}=0.41)$ were not significantly different from those of physicians $(\mathrm{M}=15.90, \mathrm{SE}=0.65)$, those for nurses $(\mathrm{M}=14.50, \mathrm{SE}=0.47)$ or those in the other category $(\mathrm{M}=14.75, \mathrm{SE}=1.38)$. Figure 1 shows a comparison of participants' self-reported confidence level regarding some core areas of the course.

Table 1. Grading Rubric for Clinical Pathway Assignment

\begin{tabular}{|c|c|c|c|c|}
\hline \multicolumn{5}{|c|}{ PERFORMANCE LEVEL } \\
\hline DESCRIPTION & Below Basic & Basic & Proficient & Advanced \\
\hline Step 1: Completion of Stakeholder Matrix /5 & $\begin{array}{l}\text { Less than } 10 \% \text { of the } \\
\text { matrix was completed }\end{array}$ & $\begin{array}{l}\text { Completed at least } \\
40 \% \text { the matrix }\end{array}$ & $\begin{array}{l}\text { Completed at least } \\
60 \% \text { of the matrix }\end{array}$ & $\begin{array}{l}\text { Completed at least } \\
80 \% \text { the matrix }\end{array}$ \\
\hline Step 2: Data to support pathway $/ 5$ & $\begin{array}{l}0-1 \text { data type and } \\
\text { source added. }\end{array}$ & $\begin{array}{l}2 \text { data types and } \\
\text { sources added, but } \\
\text { not clear }\end{array}$ & $\begin{array}{l}3 \text { data types and } \\
\text { sources identified }\end{array}$ & $\begin{array}{l}4 \text { data types and } \\
\text { sources identified }\end{array}$ \\
\hline Step 3: Develop an action plan $/ 10$ & $\begin{array}{l}\text { Identified at one } \\
\text { outcome and completed } \\
<10 \% \text { of the diagram }\end{array}$ & $\begin{array}{l}\text { Identified at } 2 \\
\text { outcomes and } \\
\text { completed at least } \\
40 \% \text { of the diagram }\end{array}$ & $\begin{array}{l}\text { Identified at } 3 \\
\text { outcomes and } \\
\text { completed at least } \\
60 \% \text { of diagram }\end{array}$ & $\begin{array}{l}\text { Identified at } 4 \\
\text { outcomes and } \\
\text { completed at least } \\
80 \% \text { of the diagram }\end{array}$ \\
\hline Steps $4 \& 5$ Dissemination and Evaluation plan /10 & $\begin{array}{l}\text { Completed }<10 \% \text { of } \\
\text { both tables }\end{array}$ & $\begin{array}{l}\text { Completed at least } \\
40 \% \text { of both tables }\end{array}$ & $\begin{array}{l}\text { Completed at least } \\
60 \% \text { of both tables }\end{array}$ & $\begin{array}{l}\text { Completed at least } \\
80 \% \text { of both tables }\end{array}$ \\
\hline
\end{tabular}


Table 2. Baseline Characteristics of Course Participants

\begin{tabular}{|c|c|}
\hline Attribute & Number $(\%)$ \\
\hline \multicolumn{2}{|l|}{ Age groups } \\
\hline$\leq 19$ years & $30(7.4)$ \\
\hline $20-29$ years & $269(65.9)$ \\
\hline $30-39$ years & $65(15.9)$ \\
\hline $40-49$ years & $22(5.4)$ \\
\hline$\geq 50$ years & $22(5.4)$ \\
\hline \multicolumn{2}{|l|}{ Sex } \\
\hline Male & $198(48.5)$ \\
\hline Female & $210(51.5)$ \\
\hline \multicolumn{2}{|l|}{ Primary practice } \\
\hline Student & $225(55.1)$ \\
\hline Physician & $90(22.1)$ \\
\hline Nurse & $77(18.9)$ \\
\hline Other & $16(3.9)$ \\
\hline \multicolumn{2}{|c|}{ Geopolitical zone of residence } \\
\hline North-central & $66(15.3)$ \\
\hline Northeast & $26(6.0)$ \\
\hline Northwest & $35(8.1)$ \\
\hline Southeast & $124(28.8)$ \\
\hline South-south & $129(29.9)$ \\
\hline Southwest & $51(11.8)$ \\
\hline \multicolumn{2}{|c|}{ Previous online course experience } \\
\hline No & $259(63.6)$ \\
\hline Yes & $148(36.4)$ \\
\hline \multicolumn{2}{|c|}{ Interval from previous course } \\
\hline$<1$ year & $105(66.5)$ \\
\hline $1-3$ years & $38(24.1)$ \\
\hline $4-5$ years & $9(5.7)$ \\
\hline$\geq 6$ years & $6(3.8)$ \\
\hline \multicolumn{2}{|c|}{ Course completion } \\
\hline Online only & $318(77.9)$ \\
\hline Blended & $90(22.1)$ \\
\hline
\end{tabular}

\section{RQ2: Competence in Clinical Pathway Development}

To address this research question, data were obtained from both online self-evaluation and the results of group work conducted during the face-to-face workshops which held in Aba, Benin, and Obong Ntak. Participants were assigned to work in multidisciplinary groups to develop local clinical pathways using a worksheet that was provided by a course instructor. The expectation was for the participants to use knowledge from the online course and of their local context to develop a flowchart for managing breast cancer. Each group's activity was graded using a rubric that focused on different competencies relevant to developing and implementing a clinical pathway, namely; stakeholder matrix, relevant data, action plan, and evaluation plan. The group scores on developing clinical pathways ranged from 63\% (19/30; Obong Ntak) to $86 \%(26 / 30$; Aba). Table 4 provides the

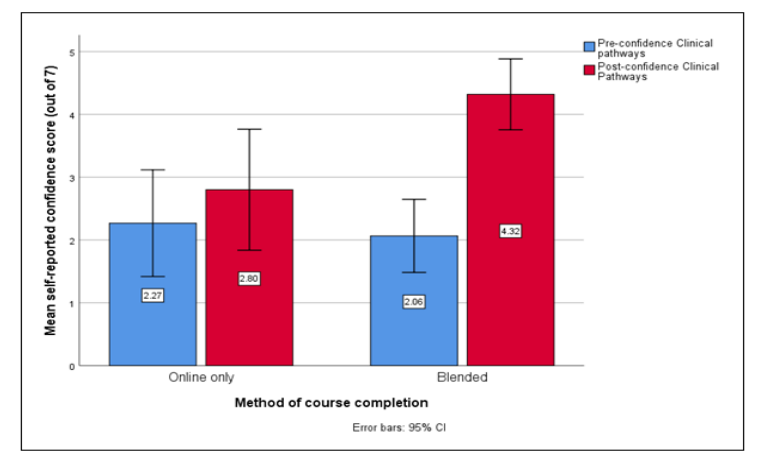

Figure 2. Comparing Self-reported Confidence Score in Developing Clinical Pathways

aggregate scores on the group activities by site (Aba, Benin, and Obong Ntak).

\section{Discussion}

Clinical pathways have been shown to improve outcomes for breast cancer patients. However, there has been no didactic course that aims to teach the skills relevant to creating local clinical pathways, prior to this course. This research is the first to report the use of this methodology for teaching the development of clinical pathways. Results show that participants improved their content knowledge relevant to NCCN breast cancer guidelines through the online course. There was a minimum of four points increase between scores in the pre and post-tests, across all participant groups. These suggest that using online teaching methods and in-person workshops can improve clinicians' knowledge and competence, respectively. It is interesting to note that individuals who completed the course using the blended format reported a significantly greater improvement in the skills regarding the development of clinical pathways (Figure 2).

The results provide empirical evidence that blended learning approach improved participants' knowledge regarding NCCN breast cancer guidelines regarding clinical pathway development. The results, however, did not reveal differences among the sub-groups with regards to their benefit from the training. This would suggest that this training approach can be equally effective for the training of medical students, nurses, physicians, and other medical professionals (Table 4). Such results have also been found in several clinical areas including

Table 3. Participants' Pretest and Post Test Scores on Breast Cancer Knowledge

\begin{tabular}{lcc}
\hline & Pretest Score & Post Test Score \\
\hline Participants' Roles & Mean $( \pm$ SD $)$ & Mean $( \pm$ SD $)$ \\
Students & $12.22( \pm 3.30)$ & $17.57( \pm 1.93)$ \\
Physicians & $13.89( \pm 1.76)$ & $17.89( \pm 1.05)$ \\
Nurses & $11.94( \pm 2.51)$ & $17.06( \pm 1.95)$ \\
Other & $13.00( \pm 2.83)$ & $16.50( \pm 0.71)$ \\
Total $(\mathrm{n}=51)$ & $12.45( \pm 2.82)$ & $17.41( \pm 1.78)$ \\
\hline
\end{tabular}


Table 4. Aggregate Scores from Clinical Pathway Group Assignments

\begin{tabular}{lccc}
\hline Step & Aba & Obong Ntak & Benin City \\
\hline Step 1: Completion of Stakeholder Matrix /5 & 3 & 2 & 3 \\
Step 2: Data to support pathway /5 & 4 & 3 & 4 \\
Step 3: Develop an action plan /10 & 9 & 7 & 8 \\
Steps 4 \& 5: Dissemination and Evaluation plan /10 & 9 & 7 & 8 \\
Total Group Score /30 & 26 & 19 & 23 \\
\hline
\end{tabular}

family medicine, orthopedics and undergraduate medical education $[9,11,15,17]$.

Furthermore, out of a maximum of 30 points, participants at the workshops scored more than $60 \%$ on the rubric used in grading competence for developing clinical pathways. Small group learning activities are usually effective for building interdisciplinary collaboration and working through complex tasks, such as developing clinical pathways [2]. Interestingly, majority of the course participants $(63 \%)$ lacked exposure to online learning prior to the course. This reflects what has been reported in extant literature about the limited utilization of online education methods in Nigeria [18-19].

This study is limited by the fact that not all participants completed all the quizzes. Although 408 people enrolled for the course and took many of the quizzes, only 51 participants completed all the quizzes. This affected the numbers that were used to calculate the effect of the online course component, although the number satisfied the criteria for statistical analysis. Meanwhile, self-reported evaluation by participants yielded results that were like the subgroup analysis, which showed overall improvement in competence. It is important that future courses would explore ways to encourage more participants to complete course quizzes. The use of conditional access restrictions which is found on Moodle learning management system but missing in Google Classroom can be helpful in this regard [20]. Due to the design of the in-person workshops (i.e. group activity), the local clinical pathway artifacts were not evaluated at the individual level. It is therefore important for future designs of similar intervention to explore ways of addressing such limitations.

In conclusion, this study has shown that online training as well as face-to-face workshops can be effective methods of teaching healthcare providers about breast cancer CPs. Participants have demonstrated their improved competence regarding breast cancer diagnosis, management as well as the development of local clinical pathways from complex guidelines. This study has sparked the interest of many organizations regarding the use of these strategies in Nigeria and elsewhere. Findings from this study will be useful in expanding the field of medical education, especially regarding training on the development of clinical pathways and use of NCCN guidelines.

\section{Acknowledgements}

This study was funded through a grant from Pfizer Inc. The funders had no role in the design, implementation, or interpretation of research findings. The authors acknowledge the contribution of study partners including Obong University, Department of Nursing Science University of Benin, Abia State Primary Health Care Development Agency

Previous presentation

American Society of Clinical Oncology, 2019 Annual Meeting

\section{References}

1. Zon RT, Frame JN, Neuss MN, Page RD, Wollins DS, Stranne S, Bosserman LD. American Society of Clinical Oncology Policy Statement on Clinical Pathways in Oncology. Journal of Oncology Practice. 2016 03;12(3):261-266. https://doi. org/10.1200/jop.2015.009134

2. Pittathankal A, Davidson T. Care pathways for patients with breast cancer. Trends in Urology, Gynaecology \& Sexual Health. 2010 03;15(2):10-13. https://doi.org/10.1002/tre.144

3. Cui Q, Tian J, Song X, Yang K, Zhang P. Effectiveness of Clinical Pathway in Breast Cancer Patients: A MetaAnalysis. Global Journal of Oncologist. 2014;2(1):15-21.

4. van Dam PA, Verheyden G, Sugihara A, Trinh XB, Van Der Mussele H, Wuyts H, Verkinderen L, Hauspy J, Vermeulen P, Dirix L. A dynamic clinical pathway for the treatment of patients with early breast cancer is a tool for better cancer care: implementation and prospective analysis between 2002-2010. World Journal of Surgical Oncology. 201303 16;11(1). https://doi.org/10.1186/1477-7819-11-70

5. Eguzo K, Camazine B. Beyond Limitations: Practical Strategies for Improving Cancer Care in Nigeria. Asian Pacific Journal of Cancer Prevention. 2013 05 30;14(5):3363-3368. https:// doi.org/10.7314/apjcp.2013.14.5.3363

6. Ekanem U, Eguzo K, Akwaowo C, Kremzier M, Eyo C, Abraham E. Cancer Education in Nigeria: Findings from a Community-based Intervention by a Physicians' Association. Cancer and Oncology Research. 2016 Dec;4(4):73-77. https://doi.org/10.13189/cor.2016.040403

7. Eguzo K, Kremzier M, Nnah K, et al. Expanding the Role of Nigerian Primary Care Providers in Cancer Control through Continuing Education: Findings from Government-led Collaborative Intervention. Clin Res Oncol. 2018;1(2):1-6.

8. Arving C, Wadensten B, Johansson B. Registered Nurses' Thoughts on Blended Learning in a Postgraduate Course in Cancer care-Content Analyses of Web Surveys and a Focus Group Interview. Journal of Cancer Education. 2013 Dec 12;29(2):278-283. https://doi.org/10.1007/s13187013-0594-6

9. Surian A, Di Masi D. Supporting Higher Education-Hospital Transition through Blended Learning. Frontiers in Education. 2017 Nov 13;2. https://doi.org/10.3389/feduc.2017.00060

10. Magaña-Valladares L, González-Robledo MC, RosasMagallanes C, Mejía-Arias MÁ, Arreola-Ornelas H, Knaul 
FM. Training Primary Health Professionals in Breast Cancer Prevention: Evidence and Experience from Mexico. Journal of Cancer Education. 201606 30;33(1):160-166. https://doi. org/10.1007/s13187-016-1065-7

11. Makhdoom N, Khoshhal KI, Algaidi S, Heissam K, Zolaly MA. 'Blended learning' as an effective teaching and learning strategy in clinical medicine: a comparative cross-sectional university-based study. Journal of Taibah University Medical Sciences. 2013 04;8(1):12-17. https://doi.org/10.1016/j. jtumed.2013.01.002

12. Cohen J. Statistical Power Analysis for the Behavioral Sciences. 2nd ed. New York: Routledge; 2013.

13. Shaw T. Cancer Care Pathways: Mapping and Dissemination Toolkit: Cancer Care Pathways for the Diagnosis of Specific Cancer Types. Cancer Institute NSW, 2018.

14. Eguzo K, Ismaila N, Oluoha C, Akpanudo U, Lawal A, Udoekong M, Jacob A, Adisa C. Using Blended-learning Approach to Improve Education on Clinical Pathways for Breast Cancer Management in Nigeria: A Research Protocol. J Clin Res Oncol. 2018;1(2):1-5.

15. Nwogu C, Ezeome E, Mahoney M, Okoye I, Michalek A. Regional Cancer Control in South-Eastern Nigeria: A Proposal Emanating from a UICC-sponsored Workshop. West African Journal of Medicine. 201109 29;29(6). https:// doi.org/10.4314/wajm.v29i6.68278

16. Meo SA. Basic steps in establishing effective small group teaching sessions in medical schools. Pakistan Journal of Medical Sciences. 201306 10;29(4). https://doi. org/10.12669/pjms.294.3609

17. Back DA, Haberstroh N, Antolic A, Sostmann K, Schmidmaier G, Hoff E. Blended learning approach improves teaching in a problem-based learning environment in orthopedics - a pilot study. BMC Medical Education. 201401 27;14(1). https://doi.org/10.1186/1472-6920-14-17

18. Eze SC, Chinedu-Eze VC, Bello AO. The utilisation of e-learning facilities in the educational delivery system of Nigeria: a study of M-University. International Journal of Educational Technology in Higher Education. 201809 19;15(1). https://doi.org/10.1186/s41239-018-0116-z

19. Eteng U, Ntui I. Access to E-learning in the Nigerian university system (NUS): a case study of University of Calabar. Information Technologist (The). 201003 12;6(2). https://doi.org/10.4314/ict.v6i2.52694

20. Moodle D. Conditional activities setting [Internet]. West Perth: Moodle; 2020 [cited 2021 Mar 21]. Available from: https://docs.moodle.org/27/en/Conditional_activities_ settings.

\section{(ब) 19}

This work is licensed under a Creative Commons AttributionNon Commercial 4.0 International License. 